\title{
Reality Cheque: Different Methods of Eliciting Fear-Related Beliefs Reveal Multiple Representations of Threat
}

James W. B. Elsey, Vivian N. Metselaar, Elias Geiser, Donna F. Knoop,

Suraya M. Gangadien, Nella A. Schrijver, Lena M. van den Nieuwenhof, Véra M. Spiekman, Marta J. Jakschik, Casper M. Enkelaar, Esperanza S. J. Visbeek, Marieke Effting, and Merel Kindt

University of Amsterdam, Department of Clinical Psychology, Amsterdam, Netherlands.

\section{Author Note}

We have no conflicts of interest to disclose. This work was supported by salaries from the University of Amsterdam and the ERC Advanced Grant 743263 of Merel Kindt.

Correspondence concerning this article should be address to James W. B. Elsey, University of Amsterdam, Postbus 15933, 1001 NK, Amsterdam, j.w.b.elsey@uva.nl 


\begin{abstract}
Influential models of fears and phobias suggest that irrational threat beliefs underpin excessive fear. Yet, many fearful individuals recognize their fear is not justified. Drawing on memory competition/multiple representations theory, we investigated whether different methods of probing fear-related beliefs might reveal conflicting representations of threat. In a proof-of-concept questionnaire study $(\mathrm{N}=145)$ and three experiments (Experiment $1, \mathrm{~N}=49$, Experiment 2, $N=47$, Experiment 3, $N=75$ ), fearful and non-fearful participants not only provided Probability Ratings for fear-related outcomes in a fear-relevant exposure task, but placed Bets, with payoffs depending on what happened in reality. Fearful participants displayed much higher Probability Ratings than Low fear participants. However, Bets revealed far less consistent group differences, even when proximal to threat (Experiments 1 and 2), and differences between High and Low fear participants' Bets disappeared when they could not be anchored to their Probability Ratings (Experiment 3). A Neutral Betting task also showed that general betting strategies were comparable between groups. Our findings are consistent with multiple representations theories of beliefs, and may provide insights into the complex interplay of adaptive and maladaptive beliefs that is a central focus of currently dominant therapies.
\end{abstract}

Keywords: Beliefs, fear, specific phobia, multiple representations, betting 


\section{FEAR-RELATED BELIEFS}

Maladaptive beliefs are a central focus of currently dominant cognitive and cognitivebehavioral approaches to understanding and treating anxiety disorders, including specific phobias (Beck et al., 2005). The core foci of such approaches vary, but a significant number which we refer to as 'threat-belief-based' models - suggest that irrational beliefs about threats posed by phobic stimuli underpin phobic anxiety (Hofmann, 2008; Smits et al., 2012). For example, summarizing their interpretations of experiments on the beliefs of spider phobic participants, Thorpe and Salkovskis (Thorpe \& Salkovskis, 1995, p.813) state: "Phobics have a range of negative beliefs about their phobic object", beliefs concerning harm appear "essential in the maintenance, and therefore the understanding, of the phobic anxiety state". As was more recently explained to patients undergoing a virtual reality cognitive treatment for fear of heights (Freeman et al., 2018, p. 627): "The reason we're afraid of heights is because we think something bad is going to happen. And that makes us feel anxious". Because such maladaptive beliefs are seen as a key cause of excessive anxiety and fear, clinicians are encouraged to elicit and challenge them either through dialogue (Beck et al., 2005), or by disproving them in behavioral experiments (Craske et al., 2014; Davis et al., 2012; Hofmann, 2008).

Research indicates that phobic people indeed express irrational beliefs regarding feared objects or situations (Arntz et al., 1993; Jones et al., 1996; Jones \& Menzies, 2000; Mavromoustakos et al., 2016; Thorpe \& Salkovskis, 1995). Some fearful and phobic individuals may even express quite bizarre or highly catastrophic beliefs, such as that a spider might hide and return having grown ten times as large (Arntz et al., 1993), or that one's plane is certain to crash five minutes after takeoff (Mavromoustakos et al., 2016). Endorsement of these beliefs would justify extreme fear, but corrective information should also be easily provided. Knowing - and rationally accepting - statistics regarding the likelihood of plane crashes or the impossibility of spiders mushrooming in size is rarely consoling to fearful individuals. Many fearful people acknowledge that their feared objects pose no realistic threat 


\section{FEAR-RELATED BELIEFS}

(Zimmerman et al., 2010), and in our experience phobic patients may even be frustrated at the suggestion that they really believe such things. How can the frequent expression of irrational threat-related beliefs among fearful or phobic individuals be reconciled with their often also having insight that their fears are not justified?

Some suggest that fearful individuals' threat-related beliefs are most active only when proximal to threat, at which point they lose insight (Beck et al., 2005). However, irrational threat-related beliefs appear to be endorsed even when distant from threat (Mavromoustakos et al., 2016). An alternative perspective is suggested by converging lines of thinking about the nature of belief from behavioral science, clinical theory, and contemporary philosophy. Gendler (2019) distinguishes between what she refers to as 'aliefs' and 'beliefs'. In essence, 'aliefs' are recalcitrant and emotionally laden responses that arise in response to a stimulus, even while more rational 'beliefs' may reflect endorsement of an entirely different perspective. For example, when a person balks at stepping onto a perfectly secure glass bridge, knowledge of safety is often rationally accepted and 'believed'. Nevertheless, the person 'alieves' or feels as if they are in danger at the same time. Similarly, Barnard and Teasdale (Barnard \& Teasdale, 1991; Teasdale, 1997) have made a distinction between intellectual and emotional belief, and Stott (2007) highlighted a similar idea under the term 'rational-emotional dissociation'.

These conceptualisations of different and sometimes conflicting forms of belief align with Brewin's (2006) proposal of memory competition, in which adaptive and maladaptive representations/beliefs may exist in parallel and compete for control over behavior. Different contextual and personal factors may result in different beliefs being retrieved or acted upon. We sought to investigate this by comparing fearful and non-fearful individuals using different means of eliciting threat-related beliefs. Participants in the following experiments were required to give probability ratings to indicate the extent to which they believed a range of fear-related events would occur during exposure to their fears (e.g., that a spider would bite 


\section{FEAR-RELATED BELIEFS}

them). This type of probability or likelihood rating is typically used in experiments and clinical investigations in which fear-related beliefs are assessed (Freeman et al., 2018; Mavromoustakos et al., 2016; Thorpe \& Salkovskis, 1995). In accordance with such studies, we expected High fear individuals to overestimate the probability of negative fear-related events relative to Low fear individuals. Crucially, we then used an alternative means of probing fear-related beliefs: asking participants to place bets on what would really occur, with payoffs tied to what happens in reality. This betting condition was intended to produce a more effortful and reflective thinking process, that might then reveal a more adaptive or realistic representation of threat.

While betting is merely one tool amongst many that might be used to try to elicit more reflective or adaptive cognition among fearful individuals, the rationale for using bets was simple. Betting behavior is commonly understood as representing a bettor's degree of belief in a proposition (Huber, 2009). While there is considerable philosophical debate regarding in what ways betting can be understood as exactly tracking beliefs, this approach matches a common understanding of what is meant when someone says they believe something: if one doubts another person's sincerity or confidence, they might ask them why they do not "put their money where their mouth is". Hence, betting matches a commonly understood means of revealing conflict in expressed beliefs. Moreover, adapting betting behavior to beliefs is simple enough for even young children to perform (Ruffman et al., 2001). Finally, although we could also try to make participants think more carefully by simply asking them to do so, or asking them to subsequently say 'whether they really believe' something after having given an initial probability rating, such approaches probably have greater risk of producing demand effects. A betting manipulation does not imply that a respondent should rethink their responses or that they may be wrong. Rather, thinking carefully about what will really happen is a generally appropriate strategy that participants will engage in provided that they want to 


\section{FEAR-RELATED BELIEFS}

make money. If, upon reflection, participants endorse their initially expressed probability ratings, then they can simply bet in accordance with them and expect to make money.

In a proof-of-concept investigation (see Appendix A), 145 participants with $(\mathrm{n}=96)$ or without $(\mathrm{n}=49)$ fear of spiders, injections, or heights provided probability ratings for events that might occur during a hypothetical fear-related situation. Compared to Low fear individuals, High fear individuals gave much higher probabilities for several fear-related negative events occurring. Participants were then led to believe that several phobic patients would take part in a real-life experiment involving essentially the same situation, and that participants could win money by accurately predicting what would happen. In this Bet condition, differences between High and Low fear participants were typically reduced or even nullified.

These preliminary findings support the idea that different means of eliciting threatrelated beliefs indeed produce different responses, with typical probability ratings revealing high expectations of threat among fearful individuals that are less strongly endorsed when they are required to place bets. However, participants gave probability ratings for themselves in the situation, then bet on what would happen with other fearful people. The findings may therefore reflect a distinction between expectations for the self vs. others. Here, we report three further behavioral experiments in which participants gave both probability ratings and bets for a real-life exposure task that they anticipated having to perform. In all experiments, betting payoffs were contingent upon accurate predictions of outcomes that were objectively verifiable and outside the control of the experimenter. To win the most money, participants would therefore need to bet on what they thought would actually happen, not just what they felt like, nor what they thought the experimenter might want them to say.

We expected that responding with a Probability Rating was likely to elicit relatively more unreflective or emotionally-driven predictions among highly fearful individuals, translating into much higher Probabilities among High than Low fear participants - as has 


\section{FEAR-RELATED BELIEFS}

been seen in previous studies (Jones et al., 1996; Jones \& Menzies, 2000; Mavromoustakos et al., 2016; Thorpe \& Salkovskis, 1995). We expected smaller or no differences between High and Low fear participants in their Bets, as the prospect of winning money by making accurate predictions about what would really happen would incentivize participants to engage in a more deliberative and reflective thinking process, enabling the retrieval or expression of more realistic threat representations (cf. Brewin, 2006). By comparing participants responses when both distal from and proximal to threat, we could also assess whether fear-related beliefs are increased during a direct fearful confrontation, and whether this undermines a fearful individual's capacity for insight (c.f. Beck et al., 2005). If so, then there should be large differences between High and Low fear participants in both Probability Ratings and Bets when Proximal. Finally, an exploratory assessment of relationships among Probabilities, Bets, and Emotions could further elucidate the extent to which participants' Probability and Bet ratings represent a conflict: if fearful individuals fully endorse the threat-related beliefs they express in Probability Ratings, then there should be a robust positive relationship between Probabilities and Bets.

\section{Method}

\section{Materials}

\section{Baseline Questionnaires}

Participants completed several baseline psychometric questionnaires (full details in Appendix B). The Fear of Spiders Questionnaire (FSQ) (Szymanski \& O’Donohue, 1995) was used to assess fear of spiders. The Patient Health Questionnaire-9 (PHQ) (Kroenke et al., 2001) was used to determine depressive symptomatology. The Spielberger State Trait Anxiety Inventory - Trait (STAI-T) (Spielberger et al., 1970) was used to assess group differences in general anxious tendencies. The Risk Orientation Questionnaire (ROQ: Rohrmann, 2005) and a modified Fear Probability Scale (cf. Öst \& Csatlos, 2000) were used in Experiments 1 and 2 to assess possible general group differences in perceived risks/risk tolerance. In Experiment 3, 


\section{FEAR-RELATED BELIEFS}

these questionnaires were dropped in favor of the Neutral Betting Task, described below. Three additional questionnaires - the Spider Automaticity-Irrationality Scale (Mayer et al., 2000) (Exp 2), the Fearful Imagery Questionnaire (Exp 2 \& 3), and Cognitive Reflection Test (Frederick, 2005) (Exp 3) were presented at the very end of some experiments for exploratory or other research purposes, detailed in Appendix C.

\section{Experimental Tasks}

Probability Rating Task. A task involving exposure to a live spider was explained to participants (see Procedure section below). Participants were then presented with a list of five fear-related events, reflecting objective outcomes that might occur during the task (e.g., "The spider will run onto my bare feet”). Items were chosen based upon previous arachnophobia questionnaire items and studies of fear-related beliefs, experience with fear-related beliefs of patients undergoing treatment for fear of spiders, and through discussion with a registered health psychologist. The possible outcomes were additionally required to be objectively assessable and out of the control of both the participants and experimenters (i.e., we did not include outcomes that were subjective, or which could be faked). For each possible outcome, participants gave a probability rating, from $0-100$, where $0=$ "Certainly will not happen", 100 $=$ "Certainly will happen", and $50=$ "Equally likely to happen or not". Items are presented in Appendix B. In Experiment 3, participants saw the same items but did not provide probability ratings, instead merely selecting the item they deemed most probable.

Emotion Rating Task. Participants reported how tense, anxious, and scared they were feeling from 0-100, with 0 labelled 'Not at all [tense/anxious/scared]', 50 labeled 'Moderately [tense/anxious/scared]', and 100 labeled as Extremely [tense/anxious/scared]'. These three items were averaged and used as a measure of anxiety-related emotions. The three items showed very high internal consistency when assessed both distant from and proximal to threat ( $\alpha=.96 \& .98$ respectively). In Experiment 3, the response scale changed from 0-100 to 1-7, 
FEAR-RELATED BELIEFS

to preclude anchoring of participants' Bets to their Emotion ratings. This response scale also showed good internal consistency (First rating $\alpha=.93$, Second rating $\alpha=.76$ ).

Spider Size Estimation. This task served as a further distraction/filler task in between the Probability and Betting tasks. Participants provided a spider size estimate by drawing a line on a piece of paper to indicate the perceived size of the spider from its top to bottom legs.

Betting Task. The outcome from the Probability Rating Task rated as most likely by the participant (specifically when distal from threat, in Experiment 1) was used for the Betting Task. We prompted participants to select one outcome if they rated two as equally likely. Participants were presented with the outcome and asked to allocate $€ 100$ between whether the event would or would not occur. They were informed that the amount of money that they could win would depend on a) the amount of money they place on each outcome, and b) what actually happens in reality when the exposure task is performed. Hence, if the participant spread the money 25:75 between the spider not jumping on them vs. jumping on them, then they would win $€ 25$ if the spider did not jump on them, and $€ 75$ if it did. Participants were informed that one person from each experiment would be randomly selected to win the money from their bet.

Neutral Betting Task. Participants were shown a 10x10 array of black and white marbles. Each column of 10 marbles was either white or black, and the total proportion of white:black marbles shown varied for each participant in increments of 10, from 10:90-90:10. It was explained that the participant would ultimately place the marbles in an opaque bag, shake it up, and later draw a marble. They were asked to split $€ 100$ between whether they would or would not draw a white marble from the bag. Participants were informed that one person would be randomly selected to win the money from their bet, with payment matching the amount of money they placed on the event that actually happened. The objective probability shown to each participant was determined pseudo-randomly, ensuring both groups had participants responding to every probability. 
FEAR-RELATED BELIEFS

\section{Procedure}

A schematic summary of the procedures is presented in Figure 1.

\section{Experiment 1}

Participants read an information brochure and gave informed consent. Experimenters checked whether participants reported taking any psychoactive medication or having a psychiatric diagnosis. Participants then completed the baseline questionnaires.

For Distal tasks, visual aids (Figure 2) were used to explain that in about 10 minutes, participants would perform an exposure task. An enclosure $(210 \mathrm{~cm} * 210 \mathrm{~cm})$ was set up in another room. On the enclosure's floor was a grid $(30 \mathrm{~cm} * 30 \mathrm{~cm}$ squares $)$. A spider stood in the center of this $7 \times 7$ grid, under a transparent cover. The participant would enter the enclosure barefooted, stand in a designated square adjacent to the spider, and answer some questions that would be explained to them at that time. Once the cover was removed, they would use a brush to gently touch the spider. A mark close to the end of the brush indicated where the participant must hold it, ensuring close proximity to the spider.

After this explanation, participants performed the Probability Rating, Emotion Rating, and Spider Size Estimation tasks. The experimenter selected the betting sheet corresponding to the participant's highest probability outcome. An example of how the betting payoffs would work was provided, focusing on predicting the weather. After confirming they understood the task, participants completed the Betting Task. 

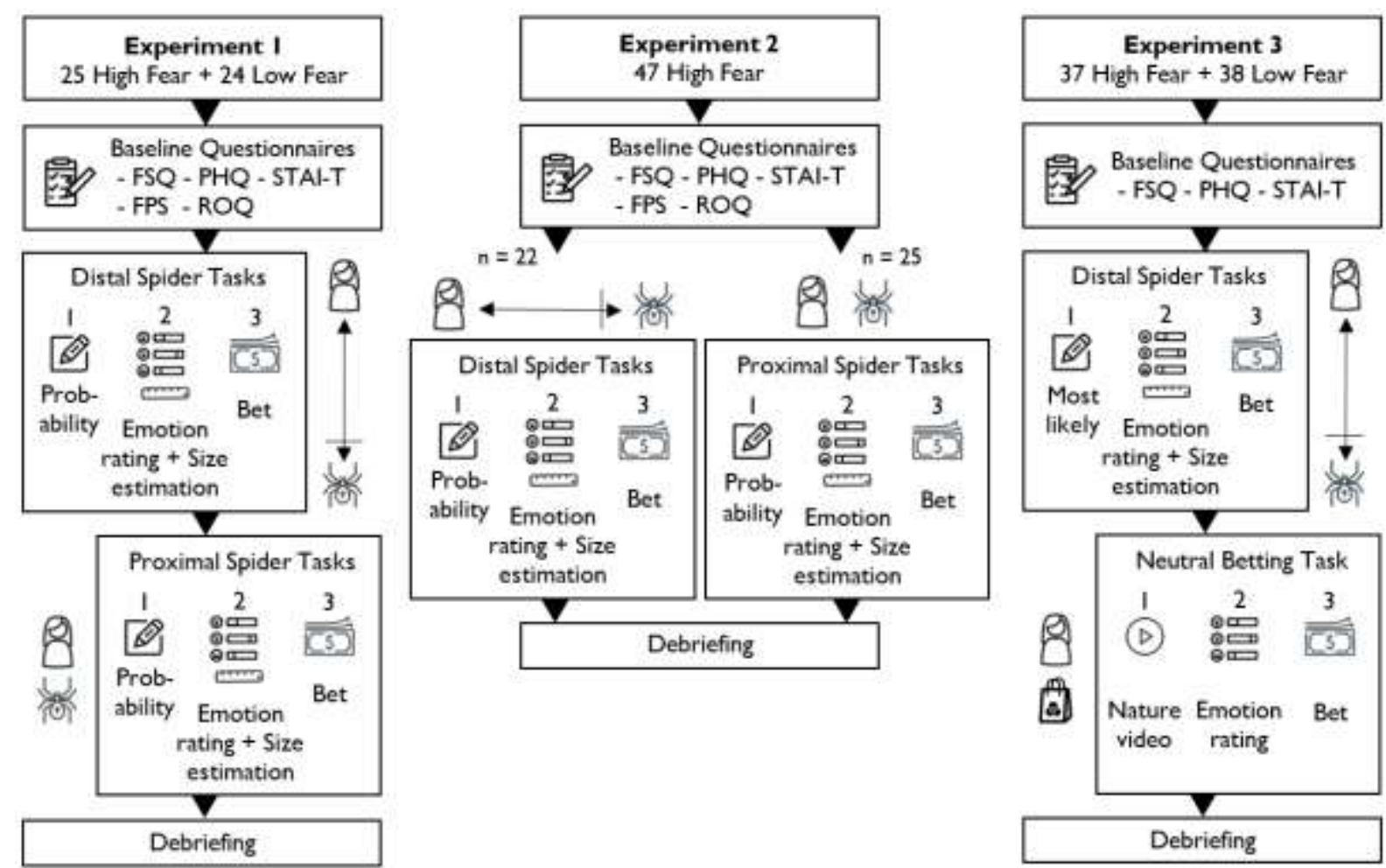

Figure 1. Procedures for Experiments 1-3.
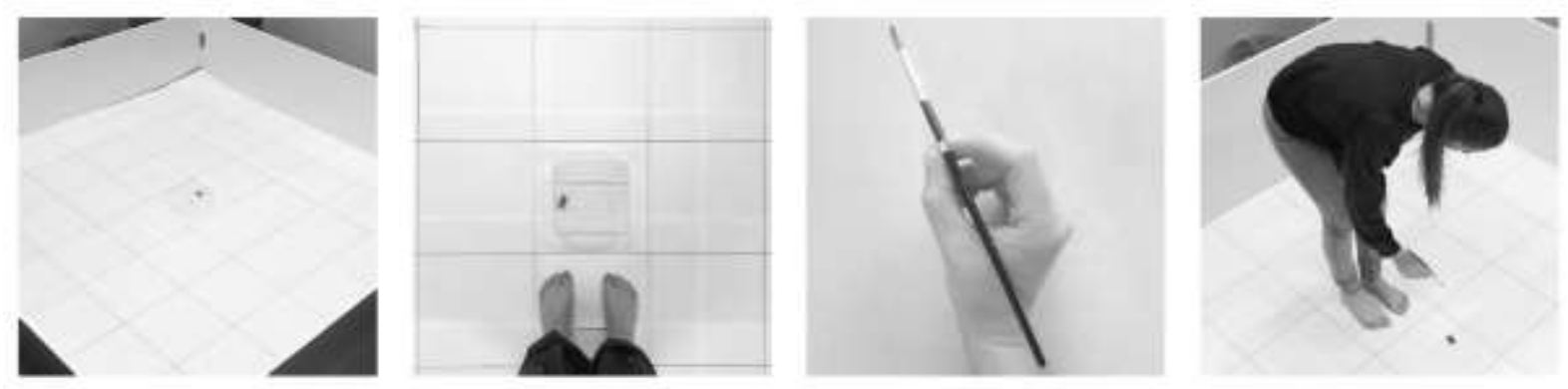

Figure 2. Pictures of the experimental setup shown to participants, depicting (from left to right) the enclosure, where the participant must stand by the spider, how they should hold the brush, and the act of reaching to touch the spider with the brush.

For Proximal tasks, we then informed participants that they would perform the exposure task. They were taken to a separate room and removed their shoes and socks before entering. In the room, the exposure task was again explained and participants entered the enclosure. The experimenter remained outside the enclosure and handed them the Probability Rating Task, Emotion Rating Task, Spider Size Estimation Task (participants briefly turned their back to the spider to make their estimate), and Betting Task in turn. To ensure 


\section{FEAR-RELATED BELIEFS}

participants were motivated to provide a true bet, it was explained that their current bets would be used to determine their payout if they were selected.

Once these Proximal tasks were performed, participants were informed that they need not undergo the exposure and could exit the enclosure. Participants completed the experiment by watching a video of what happened when someone really performed the exposure task (the spider simply runs away), and it was explained that this outcome would be used to determine their winnings. Participants were debriefed and asked to sign a form indicating that they would not discuss the research or bets/outcomes with other possible participants. Once all participants had completed the study, we randomly selected and rewarded the winning participant.

\section{Experiment 2}

As will be seen, the Proximal Probability Ratings of High fear participants in Experiment 1 could be considered surprising (they were marginally lower than Distal Probability Ratings), and we wondered whether they might have been influenced by the repeated measures design. Procedures for Experiment 2 were identical to Experiment 1, with two exceptions. Firstly, we only ran High fear participants. Secondly, the Distal and Proximal conditions were run as independent groups. Participants in the Distal condition proceeded exactly as in Experiment 1, but then did not perform the Proximal tasks. After completing baseline questionnaires, participants in the Proximal condition had the exposure task explained to them and were immediately asked if they were willing to go to the room to perform it. Once inside the exposure room they completed the Probability Rating and Emotion Rating tasks. They then briefly left the room to perform the Spider Size Estimation and had the Betting Task explained to them. This was done away from the spider to ensure complete attention to the instructions. They then returned to the square adjacent to the spider and were informed that immediately after their bet, the cover would be removed and the exposure task would commence. They were then handed the betting sheet and performed the 
FEAR-RELATED BELIEFS

Betting Task. Participants were debriefed and given the option to watch the spider video as in Experiment 1 . A winning participant was again randomly determined and rewarded once the study was completed.

\section{Experiment 3}

In Experiment 3, we considered whether the small remaining differences between High and Low fear participants' Bets observed in Experiments 1 and 2 might be due to High fear participants being biased towards or anchored by their initially very high Probability Ratings (Tversky \& Kahneman, 1974). Procedures mirrored the Distal condition in Experiment 1, with some notable exceptions. In this experiment, High and Low fear women simply picked their most-believed outcome without giving a Probability Rating, and then provided Bets, enabling the assessment of Bets without possible anchoring to initial Probability Ratings (cf. Tversky \& Kahneman, 1974). To further prevent anchoring to numbers from 0-100, participants gave Emotion Ratings on a 1-7 scale.

The ROQ and FPS were removed in favor of a direct assessment of betting in a neutral situation. After being informed they did not have to perform the exposure task, participants were informed that they could still win the promised money by betting on another situation. Participants watched a 3-minute relaxing wildlife video and provided a second Emotion Rating. Then, they performed the Neutral Betting task, in which they were shown the probability of drawing a black vs. white ball from a bag, and had to bet on what color they would draw. The purpose of this task was twofold. Firstly, we wished to show that when participants have probability information which they would endorse even upon reflection, their bets show a sensible positive relationship with that probability. The second purpose was to determine possible group differences in overall betting strategies, such as a tendency to choose risk averse bets that guarantee 50 euros, rather than responding to probabilities.

Participants were debriefed and allowed to watch the spider exposure video, and a winning participant was selected by lottery and rewarded after study completion. 
FEAR-RELATED BELIEFS

\section{Inclusion/Exclusion Criteria}

Given the disparity between men and women expressing high and low fear of spiders, we only recruited women. High fear participants were required to have FSQ scores $\geq 67$ : the top $12.5 \%$ of scores from a student sample collected in previous years (percentiles based on $\mathrm{N}$ $=315)$. The Low fear group corresponded to the bottom 50\% $(\leq 26)$. Participants were also required to be aged 18-35, score < 15 on the PHQ (the cutoff for moderately severe depression), to report an absence of diagnosed psychological disorders (besides possibly a specific phobia), and not be using psychoactive medication.

Exclusions were as follows: Other mental health problems or psychoactive medication (Experiment 1, $\mathrm{n}=3$; Experiment 3, $\mathrm{n}=1$ ), FSQ scores outside the designated ranges on the day of the experiment (Experiment 1, $\mathrm{n}=8$; Experiment 2, $\mathrm{n}=5$; Experiment 3, $\mathrm{n}=13$ ), PHQ scores too high (Experiment 1, $\mathrm{n}=5$; Experiment 2, $\mathrm{n}=3$; Experiment 3, $\mathrm{n}=4$ ). One participant dropped out in Experiment 1 due to not wishing to perform the behavioral task. In Experiment 2, one High fear participant with extensive research experience informed us that she did not believe the exposure task was real, and was therefore excluded. In Experiment 3, one participant's spider bet could not be deciphered, and was removed from analyses. Two tentative exclusions occurred due to minor experimenter errors in Experiments 2 (all participant's responses) and 3 (participant's second Emotion Rating and Neutral Bet). These two exclusions are described in Appendix D, with graphs showing negligible influence on the results.

\section{Analytic Approach}

Group comparisons on baseline measures were performed in JASP (JASP Team, 2019) using Bayesian independent samples $t$-tests and Mann-Whitney tests to ensure interpretations were robust to violations of normality. These analyses used default priors outlined in Wagenmakers et al. (2018). 
FEAR-RELATED BELIEFS

Key outcome variables - Emotion Ratings, Probabilities, Bets, and Neutral Bets were analyzed using Bayesian hierarchical 'beta regression' models in $R$ v3.5.3 (R Core Team, 2013), using RStudio v1.2.1335 (RStudio Team, 2015), and the $R$ package brms v2.12.0 (Bürkner, 2017), with repeated observations of any variable nested within subjects. All brms analyses used four Markov Chains with 8000 iterations (2000 warmup), except the Bet-Belief-Emotion multiple regressions, which used 12000 iterations (3000 warmup). Given identical response scales and equivalent procedures, data from Experiments 1 and 2 were analyzed together, enabling direct comparisons between experiments. Experiment 3 was analyzed separately owing to the different response scales/outcome variables. Following Smithson and Verkuilen (2006), responses were divided so that the highest possible rating in the outcome variable would be 1 , and the lowest 0 , producing a $0-1$ range. Data was then transformed as in Equation 1 to avoid actual zeroes or ones:

$$
y^{n}=\left[y^{\prime}(N-1)+1 / 2\right] / N
$$

This seemingly complex transformation merely 'squishes' numbers into a 0-1 range. For example, a starting response of 100 would first be transformed to 1 , and after transformation with Equation 1 might equal something like .995. For experiments 1 and 2, means ( High fearExp2), Proximity (Distal vs. Proximal from threat), and their interaction. Precision (phi of the beta distribution) was predicted by Group (Low vs. High fear - comprising all High fear participants). For some comparisons, posterior distributions for the two Distal High fear groups were averaged together (weighted according to sample size), as these groups were procedurally identical and almost exactly replicated one another (see means of $\mathrm{HD}_{1}$ and $\mathrm{HD}_{2}$ in Figure 3). A measure of distributional overlap was used (Pastore \& Calcagnì, 2019), with the $R$ package overlapping v1.6.0 (Pastore, 2018), to estimate effect size differences between groups in Probability Ratings vs. Bets. Two further exploratory regression models aimed to predict Probability Ratings from Bets and Emotions: A high fear model predicted means from 


\section{FEAR-RELATED BELIEFS}

Proximity (Distal, pooled from Experiment 1 and 2 vs. Proximalexp1 vs. ProximalExp2). A low fear model predicted means from Proximity (Distal vs. Proximal).

In Experiment 3, mean Emotion Ratings were predicted from Group (High vs. Low fear) and Timepoint ( $\mathrm{t} 1$ = anticipating spider encounter, $\mathrm{t} 2$ = before Neutral Bet $)$, and precision from Group (Low vs. High fear). Mean Bets were predicted from Group (High vs. Low fear), with the Neutral Bet model also including the objective probability of picking white, and its interaction with Group. Precision was predicted from Group (Low vs. High fear). All analyses included STAI and Age as centered predictors, due to possible confounds across conditions (see Appendix E). Possible effects of ROQ scores on outcomes in Experiments 1 and 2 were assessed and determined to be negligible in a separate regression analysis presented in Appendix F. Parameters were estimated using weakly informative priors, detailed in Appendix G.

Parameter estimates in this approach are a posterior distribution of plausible values. Hence, a point estimate (the median of the posterior distribution) is accompanied by a $95 \%$ Highest Density Interval (HDI), reflecting the $95 \%$ most probable values in the posterior, indicating uncertainty around the estimate.

Table 1a. Baseline comparisons for Experiments 1 and 2.

\begin{tabular}{|c|c|c|c|c|c|c|}
\hline & Group & Mean [SD] & Med & $\mathrm{n}$ & Test & $B F_{10}$ \\
\hline \multirow[t]{2}{*}{ Age } & Low & 19.88 [1.57] & 20.00 & 24 & $t$ & 0.88 \\
\hline & High & $20.76[2.34]$ & 20.00 & 72 & $M W$ & 0.63 \\
\hline \multirow[t]{2}{*}{ FSQ } & Low & $22.13[3.06]$ & 22.00 & 24 & $t$ & $2.21 \mathrm{E}+41$ \\
\hline & High & 91.57 [12.77] & 91.00 & 72 & $M W$ & 11086.39 \\
\hline \multirow[t]{2}{*}{ STAI-T } & Low & 39.58 [11.59] & 38.00 & 24 & $t$ & 0.27 \\
\hline & High & $40.71[9.46]$ & 39.00 & 72 & $M W$ & 0.28 \\
\hline \multirow[t]{2}{*}{ PHQ } & Low & $6.29[3.56]$ & 5.00 & 24 & $t$ & 0.34 \\
\hline & High & $5.62[3.04]$ & 5.00 & 72 & $M W$ & 0.34 \\
\hline \multirow[t]{2}{*}{ ROQ } & Low & $4.77[0.80]$ & 4.92 & 24 & $t$ & 7.86 \\
\hline & High & $4.28[0.70]$ & 4.25 & 72 & $M W$ & 6.13 \\
\hline \multirow[t]{2}{*}{ FPSs } & Low & $19.33[14.33]$ & 16.63 & 24 & $t$ & 158217.87 \\
\hline & High & $43.99[18.70]$ & 43.75 & 69 & $M W$ & 16304.28 \\
\hline \multirow[t]{2}{*}{ FPS+ } & Low & $12.18[9.40]$ & 10.60 & 24 & $t$ & 0.34 \\
\hline & High & 14.49 [11.62] & 12.40 & 69 & $M W$ & 0.30 \\
\hline \multirow[t]{2}{*}{ FPS- } & Low & $13.21[14.05]$ & 8.14 & 24 & $t$ & 0.44 \\
\hline & High & $16.93[13.35]$ & 13.43 & 69 & $M W$ & 0.85 \\
\hline
\end{tabular}


Table 1b. Baseline comparisons for Experiment 3

\begin{tabular}{ccccc|cc} 
& Group & Mean $[\mathrm{SD}]$ & Med & $\mathrm{n}$ & Test & $B F_{10}$ \\
\hline \multirow{2}{*}{ Age } & Low & $20.13[2.40]$ & 19.00 & 38 & $t$ & 0.25 \\
& High & $20.00[1.97]$ & 19.00 & 37 & $M W$ & 0.25 \\
\multirow{2}{*}{ FSQ } & Low & $2118[2.55]$ & 21.00 & 38 & $t$ & $6.36 \mathrm{E}+46$ \\
& High & $89.76[10.51]$ & 88.00 & 37 & $M W$ & 119136.11 \\
& Low & $37.82[8.61]$ & 38.00 & 38 & $t$ & 1.31 \\
& High & $42.22[10.40]$ & 42.00 & 37 & $M W$ & 0.93 \\
& Low & $4.84[2.86]$ & 4.50 & 38 & $t$ & 0.36 \\
& High & $5.60[3.82]$ & 4.00 & 37 & $M W$ & 0.33 \\
\hline
\end{tabular}

$t=$ Bayesian independent samples $t$-test; $M W=$ Bayesian Man-Whitney test;

$B F_{10}=$ Bayes factor for difference between groups; $\mathrm{SD}=$ standard deviation; Med $=$ Median

\section{Results}

\section{Experiments 1 \& 2}

Figure 3 displays posterior parameter estimates for the means of the beta distributions estimated to underpin the observed data in each group, alongside posterior distributions for several comparisons of interest between them. Plots of both means and comparisons show that Emotion ratings and Probability are highly different in Low fear vs. High fear participants $\left(\mathrm{HP}_{2}-\mathrm{LD}_{1}, \mathrm{HD}-\mathrm{LD}_{1}, \mathrm{HP}_{1}-\mathrm{LP}_{1}, \mathrm{HP}_{2}-\mathrm{LP}_{1}\right)$. Posterior estimates for comparisons between High and Low fear groups in Probabilities and Emotions show sizable and clearly non-zero differences, suggesting we can be confident that High fear participants score more highly than Low fear participants on these variables. For Bets, differences between High and Low fear participants are less clear-cut. For Distal comparisons (HD-LD 1$)$, the $95 \%$ HDI excludes 0 by a small margin. Proximal comparisons between High and Low fear participants in Experiment $1\left(\mathrm{HP}_{1}-\mathrm{LP}_{1}\right)$ indicate a tendency towards higher scores in the High fear group, but an absence of differences between groups remains plausible. Comparison of the Proximal condition for Low fear participants from Experiment 1 vs. High fear participants of Experiment $2\left(\mathrm{HP}_{2}\right.$ $\mathrm{LP}_{1}$ ) also suggests that an absence of differences between groups is well within the posterior distribution. 

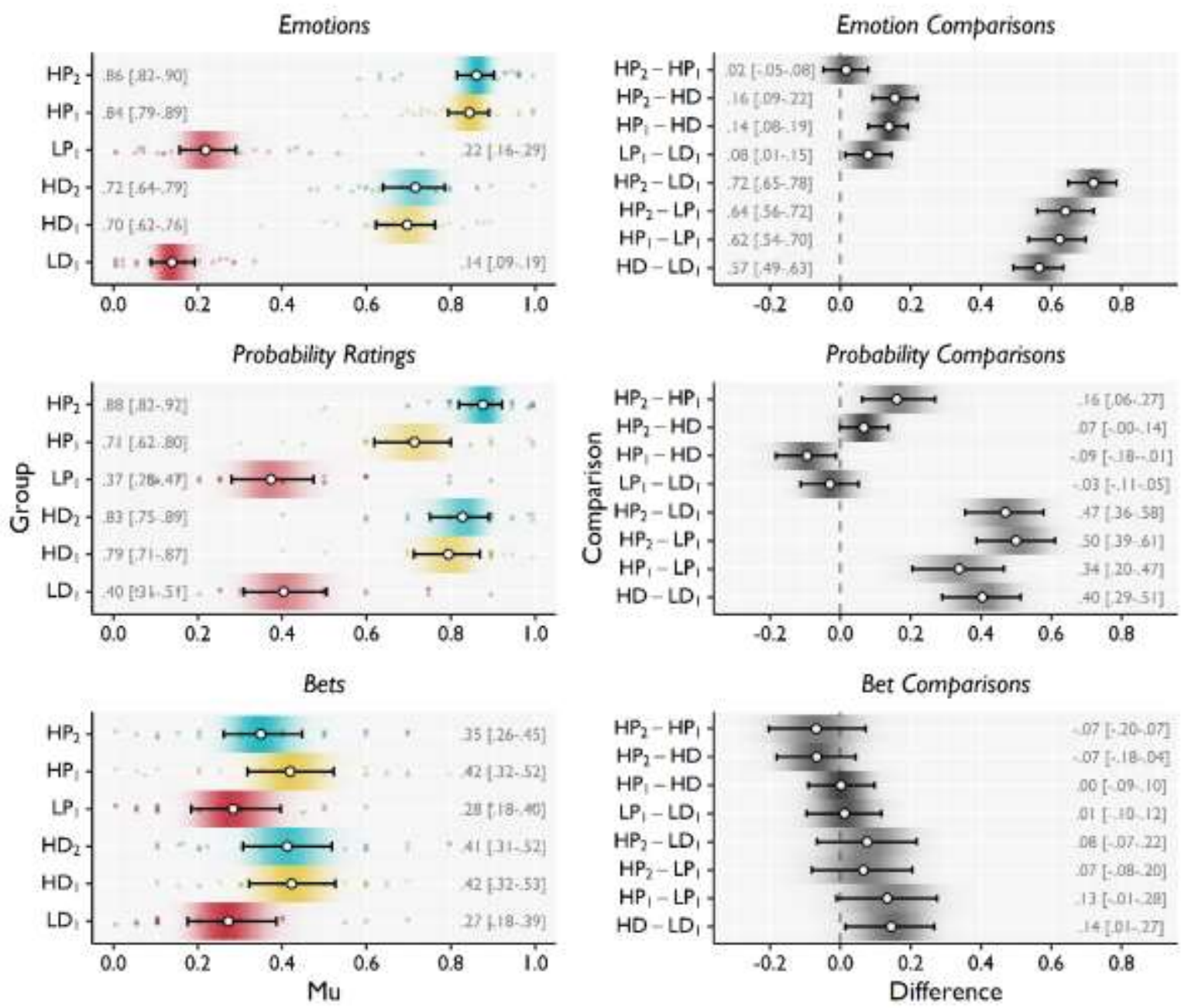

Figure 3. Posterior parameter estimates for the mean Emotion, Belief, and Bet ratings in each condition of Experiments 1 and 2 (left column), alongside several key comparisons between groups/conditions (right column). The point estimates and bars respectively represent the median and $95 \%$ HDI of the posterior estimate, also represented numerically. Shading conveys the shape of the posterior distribution, with darker shading representing higher density. Closed points represent individual responses. $\mathrm{L}=$ Low fear; $\mathrm{H}=$ High fear; $\mathrm{D}=$ Distal, $\mathrm{P}=$ Proximal, subscript 1 or $2=$ Experiments 1 or $2, \mathrm{HD}=$ combined estimate for High fear participants when Distal.

An instructive comparison can be made where we might expect to see the greatest differences between groups. It might be expected that the lowest endorsement of fear-related events occurring would be given by Low fear participants when Distal, and the highest by High fear participants placed immediately into the Proximal situation. This comparison $\left(\mathrm{HP}_{2}-\right.$ $\mathrm{LD}_{1}$ ) reveals very large differences between High and Low fear participants in their Emotions as well as Probability Ratings, yet for Bets, zero difference between groups is again well 


\section{FEAR-RELATED BELIEFS}

within the posterior distribution. The point estimate for the posterior indicates a small difference relative to the same comparison made for Probabilities or Emotions.

By incorporating estimates of the phi parameter (aka. 'precision' or 'size') of the respective beta distributions into our comparisons, we can also estimate differences in the size of group differences for Probabilities vs. Bets, using a 'distribution free overlapping index' (Pastore \& Calcagnì, 2019). This index of overlap varies from 0 (completely separated distributions) to 1 (completely overlapping distributions), and has been highlighted as a general measure for effect size when making comparisons between groups, especially when data is not normally distributed (Pastore \& Calcagnì, 2019). For the four key High vs. Low fear group comparisons discussed above, the High and Low fear groups are estimated as overlapping more greatly in their Bets than in their Probability Ratings (Figure 4), though this is a less reliable difference when Proximal responses in Experiment 1 are compared. These results support the idea that High and Low fear groups differ more in their Probability Ratings than in their Bets.

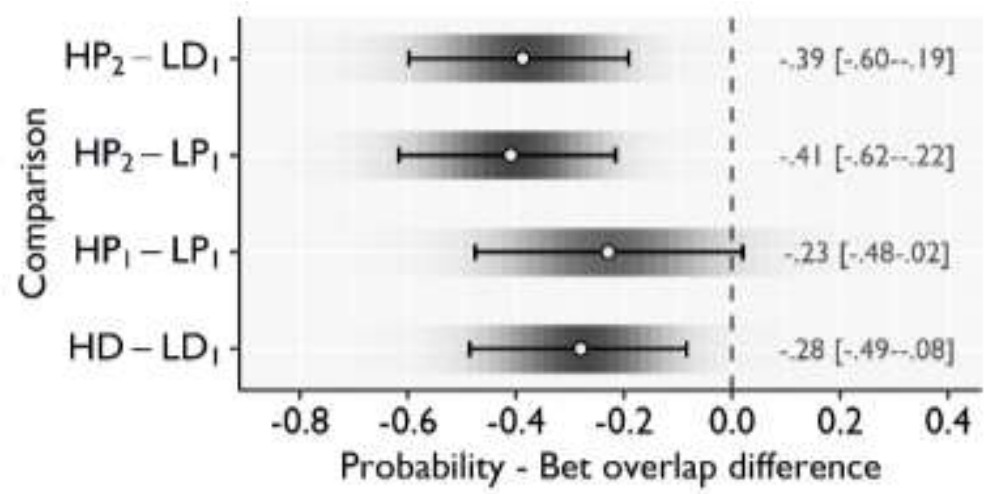

Figure 4. Difference in overlap estimates for Beliefs vs. Bets across key comparisons between High and Low fear groups. The point estimates and bars respectively represent the median and 95\% HDI of the posterior estimate, also represented numerically. Shading conveys the shape of the posterior distribution, with darker shading representing higher density. $\mathrm{L}=$ Low fear; H = High fear; D = Distal, P = Proximal, subscript 1 or $2=$ Experiments 1 or 2, HD = combined estimate for High fear participants when Distal.

Some further comparisons provide additional insight into participants' responses. Comparisons between Distal and Proximal ratings of Emotion indicate that both groups saw 


\section{FEAR-RELATED BELIEFS}

an increase in their negative emotions when Proximal ( $\left.\mathrm{LP}_{1}-\mathrm{LD}_{1}, \mathrm{HP}_{1}-\mathrm{HD}, \mathrm{HP}_{2}-\mathrm{HD}\right)$, though Low fear participants' responses remain low. Responses of High fear participants in Experiments 1 and 2 closely parallel one another, except for Proximal Probability Ratings. Under repeated measures in Experiment 1, Proximal Probability Ratings tended to be lower than Distal Probability Ratings (HP $1-H D)$. When participants went directly to the Proximal situation in Experiment 2 (HP2-HD), their Probability Ratings tended to be slightly higher than those of Distal participants. Consequently, High fear participants' Proximal Probability Ratings are higher in Experiment 2 than $1\left(\mathrm{HP}_{2}-\mathrm{HP}_{1}\right)$. Hence, Proximal assessments in Experiment 1 may have been affected by the repeated measures design. For example, performing Distal Probability Rating and Bet tasks may have enabled some participants to recognize disparities in their thinking and to change their approach to the ratings when Proximal.

This idea was examined further in two exploratory regression models (one for High fear, one for Low fear participants), investigating the associations among Emotions, Bets, and Probability Ratings. Figure 3 depicts the relationship between Bets and Probability Ratings, with Emotions held at their average (top row), and the relationship between Emotions and Probability Ratings, with Bets held at their average (middle row), for different groups and proximity levels. The bottom row compares the Emotion and Bet regression coefficients.

Among High fear participants when Distal (HD), Emotion ratings were positively related to Probability Ratings, whereas the posterior entertains negligible or even negative relationships between Bets and Probability Ratings. This pattern flips in the repeated measures Proximal condition $\left(\mathrm{HP}_{1}\right)$, and Bets are more positively related to Probability Ratings than are Emotions. This would appear to be an artifact of the repeated measures design: when sent directly to the Proximal condition $\left(\mathrm{HP}_{2}\right)$, High fear participants again display a positive relationship between Emotions and Probabilities and an inconclusive relationship between Bets and Probabilities. In contrast, Low Fear participants' Bets show a 


\section{FEAR-RELATED BELIEFS}

consistent positive relationship with their Probability Ratings, whether Distal or Proximal $\left(\mathrm{LD}_{1}, \mathrm{LP}_{1}\right)$. The absence of association between Probability Ratings and Bets among High fear participants suggests that fearful participants were reluctant to fully endorse their initially expressed Probability Ratings. Indeed, several High fear participants gave Probability Ratings of 100, yet none Bet all their money on the event, and many High fear participants bet less than 50 .
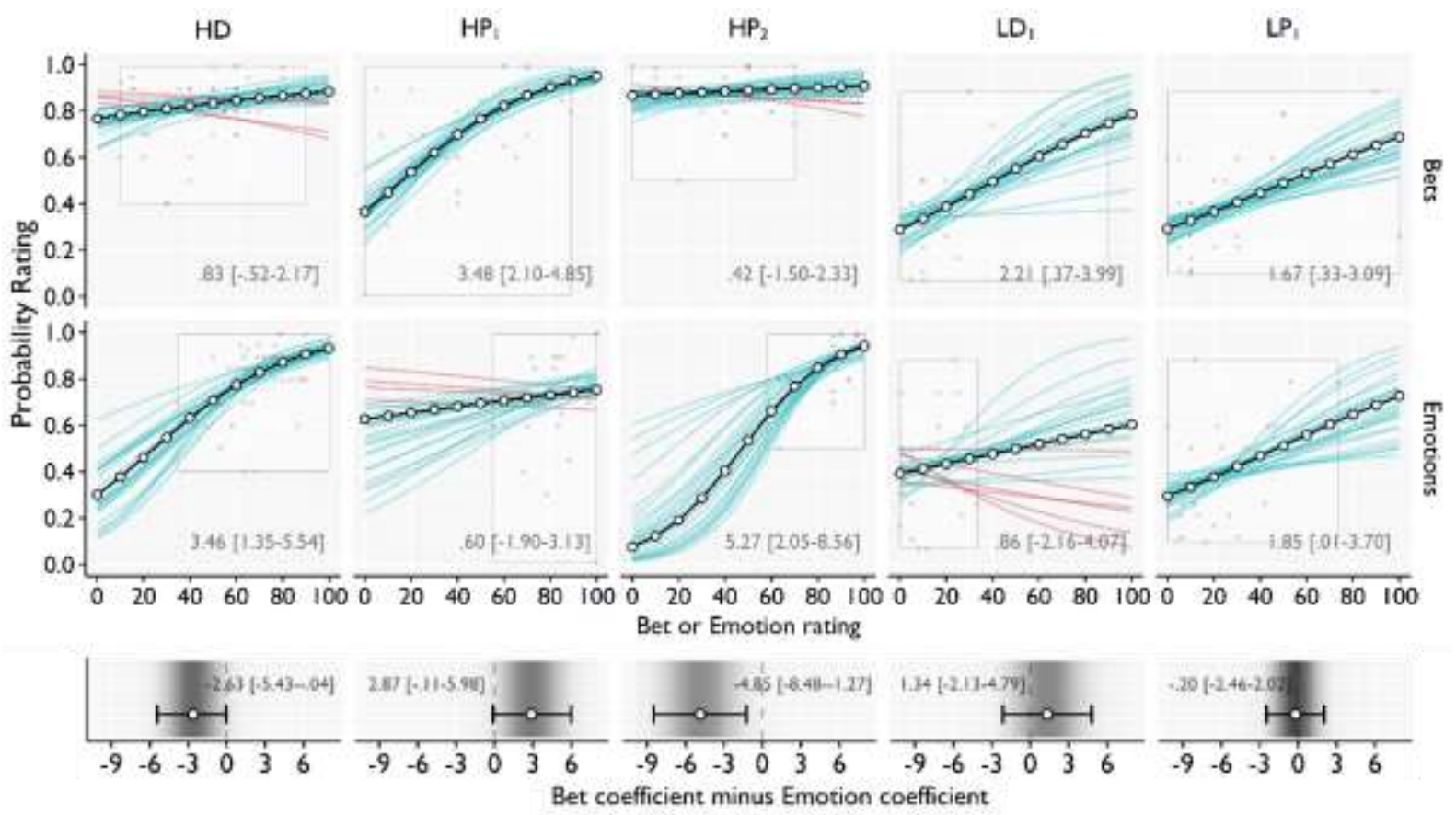

Figure 5. Conditional effects from regression models estimating Probability Ratings from Emotions and Bets. Black lines with points reflect the median estimate from the posterior distribution of Probability Ratings with varying levels of Bets (top row) or Emotions (bottom row). Colored lines represent 25 evenly-spaced draws from the posterior distribution of the respective regression coefficient. Red line $=$ Negative coefficient, Blue line $=$ positive coefficient. Boxes within each panel reflect the range of the raw data that estimates were based upon. Bottom row displays a comparison of the Bet vs. Emotion coefficient for each group/condition. The point estimates and bars respectively represent the median and 95\% HDI of the posterior estimate, also represented numerically. Shading conveys the shape of the posterior distribution, with darker shading representing higher density. $\mathrm{L}=$ Low fear; $\mathrm{H}=$ High fear; $\mathrm{D}=$ Distal, $\mathrm{P}=$ Proximal, subscript 1 or $2=$ Experiments 1 or 2 , $\mathrm{HD}=$ combined estimate for High fear participants when Distal.

\section{Experiment 3}

Figure 6 displays posterior parameter estimates for Emotions and Bets in Experiment 3, with Emotions assessed before participants believed they would encounter a spider (t1) and before providing their Neutral Bet (t2). As expected, High fear participants give much higher Emotion ratings than Low fear participants when expecting to confront a spider (HDt1-LDt1). 


\section{FEAR-RELATED BELIEFS}

Emotion ratings became much more similar before the Neutral Bet, after participants were informed that they would not have to face a spider $\left(\mathrm{HD}_{\mathrm{t} 2}-\mathrm{LD}_{\mathrm{t} 2}\right)$. Despite large differences between groups in Emotion ratings, Bets on the most-believed outcome in the spider situation were very similar, with the posterior estimate for the difference between groups evenly spanning across 0 (HD-LD), and an estimated overlap of High and Low fear Bet distributions of .74 [.57-.94].
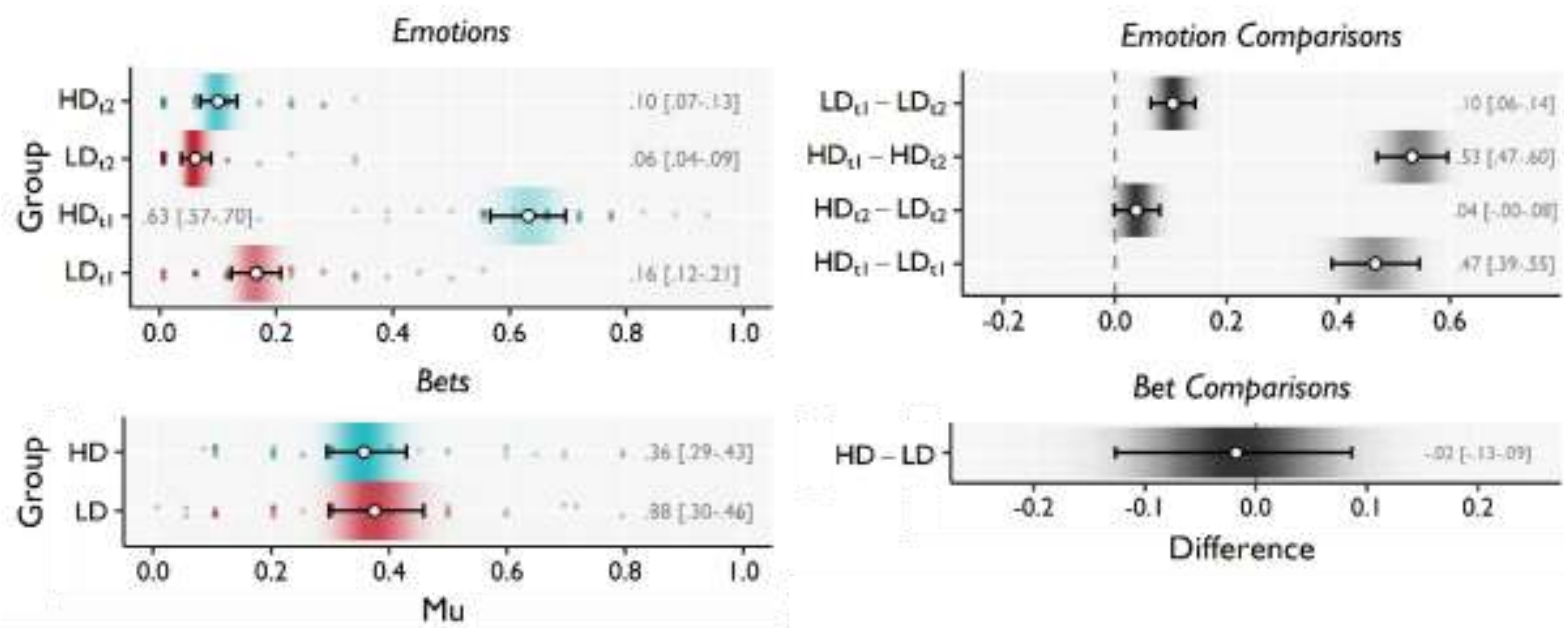

Figure 6. Posterior parameter estimates for the mean Emotion and Bet ratings in each condition of Experiment 3 (left column), alongside several key comparisons between groups/conditions (right column). The point estimates and bars respectively represent the median and $95 \%$ HDI of the posterior estimate, also represented numerically. Shading conveys the shape of the posterior distribution, with darker shading representing higher density. Closed points represent individual responses. $\mathrm{L}=$ Low fear; $\mathrm{H}=$ High fear; $\mathrm{D}=$ Distal. $\mathrm{T} 1$ and $\mathrm{T} 2$ reflect Emotion ratings before the Spider Bet and the Neutral Bet, respectively.

The above findings suggest that highly fearful individuals may not Bet in line with their initially expressed Probability Ratings for the occurrence of fear-related events, but this could reflect a general deficit in their capacity to align betting behavior with expectations, or a tendency to always favor risk averse bets rather than tracking probabilities. This was assessed by presenting participants with a Neutral Bet in which objective probabilities of the outcome were shown to participants - specifically, the objective probability of picking a white vs. black marble from a bag. Hence, even upon reflection, participants should accept the probability they were shown, as there would be no reason to doubt it, and no alternative 


\section{FEAR-RELATED BELIEFS}

representation of the probability that might be retrieved instead. Regression coefficients estimating the impact of changing the proportion of white:black marbles on Bets indicate that both Low and High fear participants bets tracked the probabilities they were shown (untransformed difference in regression coefficients: Median [95\% HDI] $=0.00$ [-.01-.02]). Hence, unlike with their expressed probabilities of fear-related events, High fear participants' Bets reliably tracked the probability of events that they had good reason to endorse. The positive regression coefficient for the effect of changing the probability of black:white balls, and the lack of differences between groups in this coefficient, also indicates that participants' strategies were not simply to try and ensure a gain of 50 euros by betting 50-50 irrespective of the probabilities, and that High fear participants showed no greater tendency to do this than Low fear participants. Though there was a slight tendency towards generally lower Bets among High vs. Low Fear participants, this was neither large nor robust.

In all analyses, posterior parameter estimates for two control variables - trait anxiety and age, spanned across zero, indicating little reliable effects attributable to them. Regression tables depicting all estimated parameters for each variable are available in Appendix $\mathrm{H}$.

\section{Discussion}

Consistent with expectations, High fear participants gave considerably higher Probabilities to fear-related events occurring than did Low fear participants. There was a slight tendency for High fear participants' probability estimates to increase when placed right in front of their feared stimulus vs. when in another room, somewhat in line with the suggestions of Beck and colleagues (Beck et al., 2005). However, large differences in Probability Ratings between High and Low fear participants were already evident when they anticipated but remained distal from the upcoming fearful encounter (Experiments 1-3), and also when merely contemplating hypothetical events (proof-of-concept study). This accords with previous findings regarding threat-related beliefs among fearful individuals (Jones et al., 1996; Jones \& Menzies, 2000; Mavromoustakos et al., 2016; Thorpe \& Salkovskis, 1995). 


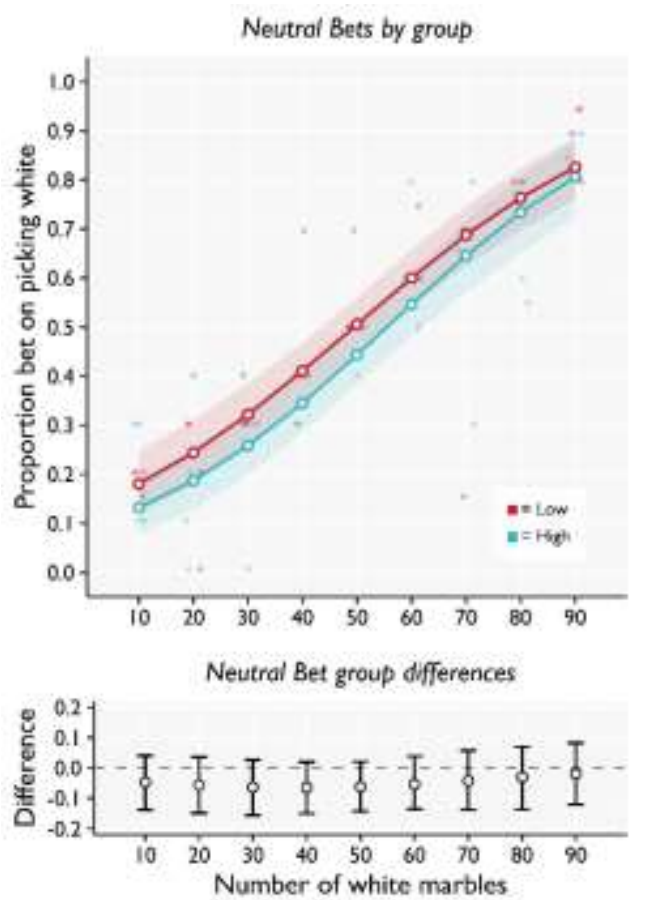

Figure 7. Conditional means for High and Low Fear participants' Neutral Bets across a range of objective probabilities (Top), along with horizontally jittered individual data points. The bottom panel displays the estimated difference between groups at each probability level, with negative estimates indicating High fear participants bet lower than Low Fear participants. Open points and error bars/ribbons respectively represent the median and 95\% HDI of the posterior distribution. Closed points represent individual responses.

Despite large differences between High and Low fear groups in Probability Ratings, group differences were smaller or absent when participants were required to Bet. When Probability and Emotion ratings could not serve as anchors for Bets (cf. Tversky \& Kahneman, 1974), High and Low fear groups bet almost identically (Experiment 3). We suggest that the prospect of winning money by making accurate predictions of what would actually happen in the situation when placing Bets may have caused participants to engage in a more reflective, effortful, or critical thinking process than when simply giving Probability Ratings. By carefully considering what was actually likely to happen in the situation, fearful participants might thus focus less on easily retrievable and emotionally provocative visions or thoughts of all the bad things that might happen and be able to retrieve and accept more realistic and adaptive representations of the situation. The Bets of High fear individuals thus 


\section{FEAR-RELATED BELIEFS}

diverge from what they might be expected to Bet if, upon reflection, they completely endorsed their initially high Probability Ratings.

Tentatively, some further support for this interpretation is provided by our exploratory analyses of the relationships among Probability Ratings, Emotion Ratings, and Bets. Low fear participants displayed a clear positive relationship between Probability Ratings and Bets. In contrast, among High fear individuals, Probability Ratings were strongly and positively correlated with Emotion Ratings, but not with Bets. Only under repeated measures conditions, when participants might have adapted their rating strategy after seeing the discrepancy between their initial Probabilities and Bets, was a relationship between Probability Ratings and Bets observed. The lack of congruence between Probabilities and Bets among High fear participants does not seem explicable on account of a general deficit in responding to probabilities, as they were equally responsive to different probabilities as Low fear participants in a Neutral Bet (Experiment 3). In the Neutral Betting Task, there is little space for conflicting representations of what is likely to happen. For the fear-related Bet, careful thinking may retrieve a whole host of different knowledge and beliefs that run counter to the initially very high Probability Ratings of fearful individuals, whereas Low fear participants may have been more likely to retrieve a realistic or adaptive representation of the situation in the first place, and therefore bet in accordance with it.

This multiple representations perspective (Brewin, 2006) may shed some light on the ostensible contradiction between fearful individuals both expressing irrational threat-related beliefs while also often acknowledging that there is little justification for their fears. Our findings suggest that exactly how a person's beliefs are probed may reveal quite different and even contradictory perspectives. Most animal phobias are already acquired by age 10 (Becker et al., 2007), meaning that for fearful adults, an emotionally laden and fearful representation of the phobic object has likely had many years to be reinforced through fearful encounters and avoidance before more reflective capabilities fully develop. These thoughts may become even 


\section{FEAR-RELATED BELIEFS}

more well-ingrained through rehearsal and simulation of catastrophic outcomes in imagination. Such a strong and well-consolidated representation may be so easily retrievable as to appear almost automatically when thinking about a feared stimulus, arriving not as a logical consideration of threat but as a strongly-felt sense of impending danger or intrusive images of imagined catastrophes. Fearful individuals thus report what they feel on the basis of this rapid threat signal, or their general feelings towards the feared object. Nevertheless, such individuals may be perfectly capable of recognizing - though perhaps with a little more effort

- that such expectations are not realistic. As highlighted by several authors (Barnard \& Teasdale, 1991; Clark, 2018; Gendler, 2019), however, this 'intellectual' recognition may be relatively less retrievable, or have lower motivational force when retrieved. Such recognition will not necessarily outweigh highly salient and easily retrievable maladaptive representations in competing for control over behavior. One possible implication of our findings is that relatively simple devices that encourage greater reflection, such as thinking whether one would place money on an event actually happening in reality (a 'reality cheque'), may aid such retrieval and help fearful individuals gain or maintain insight into the irrationality of some of their maladaptive threat-related beliefs. With retrieval aids and practice, more reflective ways of thinking may become intuitive and automatic (De Neys \& Pennycook, 2019). However, this will require further experimentation in clinically phobic populations to fully evaluate.

It may be questioned whether observed betting responses really represent endorsement of a belief, or some methodological artifact. The potential financial gain was rather high (though not certain to be received), and High fear participants may have been motivated to provide lower Bets relative to Probabilities simply to try to ensure they would win at least 50 euros. Yet, in a supplementary analysis we found that risk aversion did not predict the chance that participants would bet away from 50 euros. Many highly fearful individuals even bet below 50 euros. This is easily explained if the participants recognised that the outcome was 


\section{FEAR-RELATED BELIEFS}

less than 50\% likely. There was considerable variability in participants' bets and this may reflect a range of financial optimization strategies that they engaged in, but simply betting 5050 to minimize loss seems unlikely. Nor can the absence of associations between Probabilities and Bets among High fear individuals be attributed to Bets simply reflecting noise: Low fear participants showed a relationship between Probabilities and Bets, and High fear participants also did when given the opportunity to perform the task a second time. Furthermore, when High fear participants had good reason to believe in a certain probability - as in the neutral task - their Bets tracked the probabilities just like Low fear participants. As the experimenter had no control over the fear-related outcomes, and the betting instruction gave no indication that participants should re-appraise their initial Probability, we also do not believe that the Bets reflect a demand effect. Moreover, there is no clear reason why a demand effect would not also manifest in Probability ratings, if it affected Bets. In short, though a novel approach and subject to different optimization strategies that may produce considerable variation in responses, we believe it is reasonable to take the bets at face value, representing participants indicating what they endorse as likely to really happen.

It should be stressed that betting behaviour is just one means of assessing multiple representations. In fact, a 'probability rating' that was explained meticulously to participants in terms of the number of times an event might happen out of 100 , and to which they had to allocate counters or some other more tangible means of endorsement, might produce similar effects. Betting responses also should not strictly be taken as reflecting what fearful individuals 'really' believe. Fearful and phobic individuals clearly also engage in other behaviours that indicate endorsement of certain irrational threat-related beliefs which are in accordance with their more simply-expressed threat expectations. While avoidance of a phobic object could merely reflect the person trying to avoid something that would give them unpleasant feelings, more specific actions such as safety behaviours often relate logically to the prevention of feared outcomes (Salkovskis, 1991). Such behaviours could be seen as 


\section{FEAR-RELATED BELIEFS}

another way in which fearful individuals 'put their money where their mouth is'. We suggest that considering these many different ways in which a fearful individual may or may not endorse their beliefs, and acknowledging differences between beliefs that are merely felt to be true vs. those that are accepted even upon reflection could prove informative.

There are methodological considerations that limit the scope of our findings. Firstly, our fearful participants were not assessed for the presence of clinically significant phobias. Yet, participants' questionnaire scores were well within the ranges of phobic participants assessed in previous studies (Muris \& Merckelbach, 1996). Moreover, our findings parallel clinical observations (Stott, 2007). Nevertheless, extending our paradigm to a clinical sample is something we intend to pursue. Our behavioural experiments also only involved young women, with a focus on fear of spiders. Spider phobia is over-represented in women, but evidently also occurs in men (Muris \& Merckelbach, 2012). Although we are not aware of a reason why such phenomena should be less apparent among men, this should be tested in future research. Regarding the specificity of our findings to fear of spiders, our proof-ofconcept questionnaire study at least intimated that such discrepancies between initial Probability Ratings and Bets may also occur in situational and other object-related phobias besides arachnophobia. Research from other domains of psychology, such as in relation to superstitious belief (Risen, 2016), highlights that beliefs across a range of domains in which thoughts and feelings interact may be similarly complex. It would therefore be surprising if such discrepancies only occurred in relation to a very specific fear in very specific circumstances. However, research directly in clinical samples and with other fears is warranted to assess the generalizability of our findings.

One further aspect of generalizability concerns the selection of outcomes with which we presented participants. Choosing our own set of outcomes ensured that participants would have to select an objectively verifiable event, with an emphasis on threat-beliefs regarding the behaviour of the spider, rather than subjective responses of the participants. Our main findings 


\section{FEAR-RELATED BELIEFS}

may not extend to participants' beliefs regarding their capabilities of dealing with the situation, or how terrible they will find it. We expect participants might actually bet in line with expressed probabilities regarding such factors, and this will be interesting to investigate. Still, participants appeared to find the outcomes we presented them with highly plausible. Even if the selected outcomes do not represent an ultimate or core fear of participants, discrepancies between the strong belief expression as assessed by initial Probability Ratings vs. weaker belief expression in the form of Bets remains instructive. Our findings also speak primarily to estimates of the likelihood, rather than magnitude of aversive events, which appraisal theories rightly acknowledge as another dimension along which fearful people may diverge from their less fearful counterparts (Yih et al., 2019). Participants in general did not choose an objectively catastrophic outcome as most probable (e.g., "I will have a heart attack"). It would be interesting to find out how much participants endorse these catastrophic items, and the costs they associate with seemingly less catastrophic ones. We recognize that even if High and Low fear individuals gave the exact same Probabilities or Bets to a negative fear-related event, the fearful people would likely associate the event with a greater cost, even if just in terms of its subjective unpleasantness.

In summary, and consistent with multi-representational theories of belief (Brewin, 2006), we found that the manner in which fearful individuals' beliefs are probed can result in divergent responses. Probability ratings tended to produce responses indicating that fearful individuals believed the likelihood of negative fear-related events occurring was almost certain, and much higher than Low fear individuals. When required to Bet on the events occurring in reality, however, differences between High and Low fear groups were reduced or even nullified. We suggest that these findings may reflect the multi-representational nature of belief, in which both adaptive and maladaptive representations of a feared object may exist in one person, with personal and contextual factors determining which form of representation is retrieved or expressed. This perspective may help understand some strange phenomena 


\section{FEAR-RELATED BELIEFS}

among fearful individuals - such as the presence of irrational threat-related beliefs combined with acknowledgment that there is no reason to be afraid - and may have implications for therapy geared towards promoting adaptive beliefs, and combating maladaptive ones.

\section{References}

Arntz, A., Lavy, E., \& van Rijsoort, S. (1993). Negative beliefs of spider phobics: A psychometric evaluation of the spider phobia beliefs questionnaire. Advances in Behavior Research and Therapy, 15(4), 257-277.

https://www.sciencedirect.com/science/article/pii/014664029390012Q

Barnard, P. J., \& Teasdale, J. D. (1991). Interacting cognitive subsystems: A systemic approach to cognitive-affective interaction and change. Cognition \& Emotion, 5(1), 139. https://doi.org/10.1080/02699939108411021

Beck, A., Emery, G., \& Greenberg, R. (2005). Anxiety disorders and phobias: A cognitive perspective. Basic Books. https://psycnet.apa.org/record/2006-01301-000

Brewin, C. R. (2006). Understanding cognitive behaviour therapy: A retrieval competition account. Behaviour Research and Therapy, 44(6), 765-784. https://doi.org/10.1016/j.brat.2006.02.005

Bürkner, P.-C. (2017). brms: An R package for Bayesian multilevel models using Stan. Journal of Statistical Software, 80(1), 1-28.

Clark, D. M. (2018). Cognitive Therapy for Social Anxiety Disorder in Adults and Adolescents. University of Lousville Depression Center Annual Conference.

Craske, M. G., Treanor, M., Conway, C. C., Zbozinek, T., \& Vervliet, B. (2014). Maximizing exposure therapy: An inhibitory learning approach. Behaviour Research and Therapy, 58, 10-23. https://doi.org/10.1016/j.brat.2014.04.006

Davis, T., Ollendick, T., \& Öst, L. (2012). Intensive one-session treatment of specific phobias. Springer. https://link.springer.com/content/pdf/10.1007/978-1-4614-3253-1.pdf 
FEAR-RELATED BELIEFS

De Neys, W., \& Pennycook, G. (2019). Logic, fast and slow: Advances in dual-process theorizing. Current Directions in Psychological Science, 28(5), 503-509. https://doi.org/10.1177/0963721419855658

Frederick, S. (2005). Cognitive reflection and decision making. Journal of Economic Perspectives, 19(4), 25-42. https://doi.org/10.1257/089533005775196732

Freeman, D., Haselton, P., Freeman, J., Spanlang, B., Kishore, S., Albery, E., Denne, M., Brown, P., Slater, M., \& Nickless, A. (2018). Automated psychological therapy using immersive virtual reality for treatment of fear of heights: a single-blind, parallel-group, randomised controlled trial. The Lancet Psychiatry, 5(8), 625-632. https://www.sciencedirect.com/science/article/pii/S2215036618302268

Gendler, T. (2019). Alief and Belief. In Contemporary Epistemology (pp. 91-109). John Wiley \& Sons, Inc. https://doi.org/10.1002/9781119420828.ch7

Hofmann, S. G. (2008). Cognitive processes during fear acquisition and extinction in animals and humans: Implications for exposure therapy of anxiety disorders. Clinical Psychology Review, 28(2), 199-210. https://www.sciencedirect.com/science/article/pii/S0272735807000979

Huber, F. (2009). Belief and Degrees of Belief. In Degrees of Belief (pp. 1-33). Springer. https://doi.org/10.1007/978-1-4020-9198-8_1

JASP Team. (2019). JASP (0.9.2).

Jones, M. K., \& Menzies, R. G. (2000). Danger expectancies, self-efficacy and insight in spider phobia. Behaviour Research and Therapy, 38(6), 585-600. https://www.sciencedirect.com/science/article/pii/S0005796799000765

Jones, M. K., Whitmont, S., \& Menzies, R. G. (1996). Danger expectancies and insight in spider phobia. Anxiety, 2(4), 179-185. https://doi.org/10.1002/(SICI)15227154(1996)2:4<179::AID-ANXI4>3.0.CO;2-J

Kroenke, K., Spitzer, R. L., \& Williams, J. B. W. (2001). The PHQ-9: validity of a brief 
FEAR-RELATED BELIEFS

depression severity measure. Journal of General Internal Medicine, 16(9), 606-613. https://doi.org/10.1046/j.1525-1497.2001.016009606.x

Mavromoustakos, E., Clark, G. I., \& Rock, A. J. (2016). Evaluating perceived probability of threat-relevant outcomes and temporal orientation in flying phobia. PLOS ONE, 11(8), e0161272. https://doi.org/10.1371/journal.pone.0161272

Mayer, B., Merckelbach, H., \& Muris, P. (2000). Self-Reported Automaticity and Irrationality in Spider Phobia. Psychological Reports, 87(2), 395-405. https://doi.org/10.2466/pr0.2000.87.2.395

Muris, P., \& Merckelbach, H. (1996). A comparison of two spider fear questionnaires. Journal of Behavior Therapy and Experimental Psychiatry, 27(3), 241-244. https://www.sciencedirect.com/science/article/pii/S0005791696000225

Muris, P., \& Merckelbach, H. (2012). Specific Phobia: Phenomenology, Epidemiology, and Etiology. In T. Davis, T. Ollendick, \& L. Öst (Eds.), Intensive one-session treatment of specific phobias (pp. 3-18). Springer. https://doi.org/10.1007/978-1-4614-3253-1_1

Öst, L., \& Csatlos, P. (2000). Probability ratings in claustrophobic patients and normal controls. Behaviour Research and Therapy, 38(11), 1107-1116. https://www.sciencedirect.com/science/article/pii/S0005796799001515

Pastore, M. (2018). Overlapping: a R package for estimating overlapping in empirical distributions. Journal of Open Source Software, 32(1023).

Pastore, M., \& Calcagnì, A. (2019). Measuring distribution similarities between samples: A distribution-free overlapping index. Frontiers in Psychology, 10. https://doi.org/10.3389/fpsyg.2019.01089

R Core Team. (2013). R: A language environment for statistical computing (3.5.3).

Risen, J. L. (2016). Believing what we do not believe: Acquiescence to superstitious beliefs and other powerful intuitions. Psychological Review, 123(2), 182-207. https://doi.org/10.1037/rev0000017 
FEAR-RELATED BELIEFS

Rohrmann. (2005). Risk attitude scales: concepts, questionnaires, utilizations. In rohrmannresearch.net. http://rohrmannresearch.net/pdfs/rohrmann-racreport.pdf

RStudio Team. (2015). RStudio: intergrated development for $R$ (1.2.1335). RStudio Inc.

Ruffman, T., Garnham, W., Import, A., \& Connolly, D. (2001). Does eye gaze indicate implicit knowledge of false belief? Charting transitions in knowledge. Journal of Experimental Child Psychology, 80(3), 201-224. https://doi.org/10.1

Salkovskis, P. M. (1991). The importance of behaviour in the maintenance of anxiety and panic: A cognitive account. Behavioural and Cognitive Psychotherapy, 19(1), 6-19. https://www.cambridge.org/core/journals/behavioural-and-cognitivepsychotherapy/article/importance-of-behaviour-in-the-maintenance-of-anxiety-andpanic-a-cognitive-account/B3BAA0EFEF179C99BBCA5F983B05A534

Smithson, M., \& Verkuilen, J. (2006). A better lemon squeezer? Maximum-likelihood regression with beta-distributed dependent variables. Psychological Methods, 11(1), 5471.

Smits, J. A. J., Julian, K., Rosenfield, D., \& Powers, M. (2012). Threat reappraisal as a mediator of symptom change in cognitive-behavioral treatment of anxiety disorders: A systematic review. Journal of Consulting and Clinical Psychology, 80(4), 624-635. https://psycnet.apa.org/record/2012-15541-001

Spielberger, C., Gorsuch, R., \& Lusthene, R. (1970). Manual for the State-Trait Anxiety Inventory. Consulting Psychologists.

Stott, R. (2007). When head and heart do not agree: a theoretical and clinical analysis of rational-emotional dissociation (RED) in cognitive therapy. Journal of Cognitive Psychotherapy, 21(1), 37-50.

http://search.proquest.com/openview/e2cab288f51130d57f926c60ab6eb384/1?pqorigsite $=$ gscholar $\&$ cbl $=28723$

Szymanski, J., \& O’Donohue, W. (1995). Fear of spiders questionnaire. Journal of Behavior 
FEAR-RELATED BELIEFS

Therapy and Experimental Psychiatry, 26(1), 31-34.

https://www.sciencedirect.com/science/article/pii/000579169400072T

Teasdale, J. D. (1997). The transformation of meaning: The Interacting Cognitive Subsystems approach. In M. J. Power \& C. R. Brewin (Eds.), The transformation of meaning in psychological therapies: Integrating theories and practice. John Wiley \& Sons, Inc. https://psycnet.apa.org/record/1997-08945-008

Thorpe, S., \& Salkovskis, P. (1995). Phobic beliefs: Do cognitive factors play a role in specific phobias? Behavior Research and Therapy, 33(7), 805-816. https://www.sciencedirect.com/science/article/pii/000579679500022P

Tversky, A., \& Kahneman, D. (1974). Judgment under uncertainty: Heuristics and biases. Science, 185(4157), 1124-1131.

https://science.sciencemag.org/content/185/4157/1124.short

Wagenmakers, E.-J., Love, J., Marsman, M., Jamil, T., Ly, A., Verhagen, J., Selker, R., Gronau, Q. F., Dropmann, D., Boutin, B., Meerhoff, F., Knight, P., Raj, A., van Kesteren, E.-J., van Doorn, J., Šmíra, M., Epskamp, S., Etz, A., Matzke, D., .. Morey, R. D. (2018). Bayesian inference for psychology. Part II: Example applications with JASP. Psychonomic Bulletin \& Review, 25(1), 58-76. https://doi.org/10.3758/s13423017-1323-7

Yih, J., Uusberg, A., Taxer, J. L., \& Gross, J. (2019). Better together: a unified perspective on appraisal and emotion regulation. Cognition \& Emotion, 33(1), 41-47.

Zimmerman, M., Dalrymple, K., Chelminski, I., Young, D., \& Galione, J. N. (2010). Recognition of irrationality of fear and the diagnosis of social anxiety disorder and specific phobia in adults: Implications for criteria revision in DSM-5. Depression and Anxiety, 27(11), 1044-1049. https://doi.org/10.1002/da.20716 